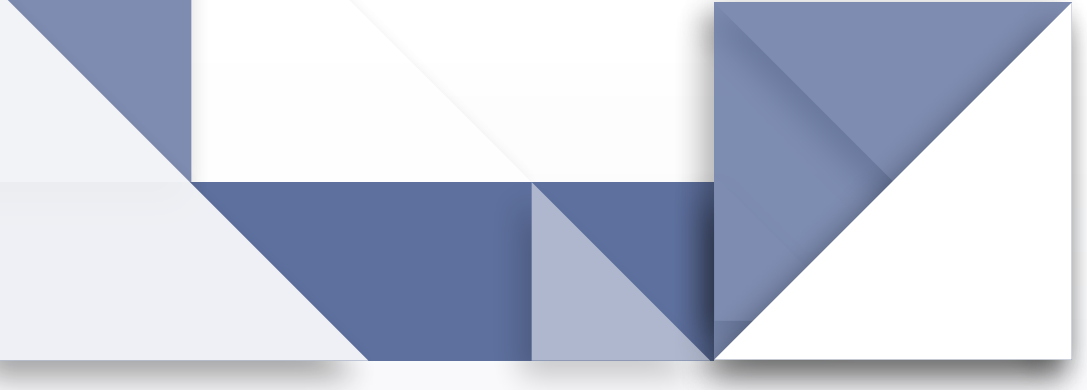

\title{
La sensibilidad ética y el conocimiento didáctico del contenido de los profesores de ciencias experimentales
}

\section{- The Ethical Sensitivity and the Pedagogical Content Knowledge of Experimental Science Teachers}

- A sensibilidade ética e conhecimento didático do conteúdo de professores de ciências experimentais

\section{Resumen}

Los constantes avances en ciencia y tecnología plantean una necesidad permanente de revisión y formación de profesores en servicio. Dentro de estas actualizaciones se debe prestar especial atención no solo a las innovaciones pedagógicas en términos de contenidos y herramientas, sino también a la sensibilidad ética; es decir, la evaluación moral de las acciones y cómo estas afectan a uno mismo y a otros. A fin de contribuir con este enfoque, se presentan los resultados de una investigación realizada desde un marco interdisciplinar que combina los modelos del Conocimiento Didáctico del Contenido (CDC), los aportes de la naturaleza de la ciencia y la interrogación sobre aspectos éticos de la enseñanza. Así, se implementó una secuencia didáctica con un grupo de profesores de ciencias argentinos en un curso de formación de posgrado empleando un recurso audiovisual para el análisis de una clase de matemáticas; las respuestas a un cuestionario se evaluaron empleando el análisis de contenido y la aplicación del test de sensibilidad ética (REST). Los resultados mostraron la capacidad de los docentes para reconocer y reflexionar sobre aspectos pedagógicos de las prácticas educativas, mientras las cuestiones éticas permanecieron en un nivel más superficial. En ese sentido, la menor importancia brindada a las cuestiones éticas alerta sobre la necesidad de la incorporación del conocimiento ético como un nuevo componente del CDC y, por ende, la formación explícita del cuerpo docente en dichos aspectos.

Palabras clave

ética; formación de docentes; investigación pedagógica; material audiovisual; responsabilidad del docente

\section{Irene Cambra Badii* María Gabriela Lorenzo**}

\footnotetext{
* Dra. en Psicología; investigadora en la Universitat de Vic-Universitat Central de Catalunya y en el Grupo de Investigación sobre Educación en Ciencias de la Salud, Universitat Pompeu Fabra, Barcelona. Correo electrónico: irene.cambra@ uvic.cat. Código Orcid: 0000-0003-1233-3243

** Dra. en Química Orgánica, Directora del Centro de Investigación y Apoyo a la Educación Científica (CIAEC), Facultad de Farmacia y Bioquímica, Universidad de Buenos Aires; investigadora independiente de la carrera del Investigador Científico del Conicet, Argentina. Correo electrónico: glorenzoffyb@gmail.com. Código Orcid: 0000-0002-9957-8392
} 


\section{Abstract}

The constant advances in science and technology pose a persistent need for revision and training of teachers in service. Within these updates, special attention should be paid not only to pedagogical innovations in terms of content and tools, but also to ethical sensitivity, that is, the moral evaluation of actions and how they affect oneself and others. To contribute with this approach, we present the results of a research carried out from an interdisciplinary framework that combines the models of the Pedagogical Knowledge of Content (PCK), the contributions of the Nature of Science and the interrogation on ethical aspects of teaching. A didactic sequence was implemented with a group of Argentine science teachers in a postgraduate training course using an audiovisual format for the analysis of a mathematics class. The responses to a questionnaire were evaluated using content analysis and the application of the ethical sensitivity test (REST). The results showed the ability of teachers to recognize and reflect on pedagogical aspects of educational practices, while ethical issues remained at a more superficial level. The minor importance given to ethical issues alerts us to the need for the incorporation of ethical knowledge as a new component of the CDC, and therefore, the need of teacher training in these aspects.

Keywords

audiovisual materials; educational research; ethics; teacher education; teacher responsibility

\section{Resumo}

Os constantes avanços em ciência e tecnologia impõem uma necessidade permanente de revisão e capacitação de professores em serviço. Dentro destas atualizações, atenção especial deve ser dada não apenas às inovações pedagógicas em termos de conteúdo e ferramentas, mas também à sensibilidade ética; —ou seja, a avaliação moral das ações e como elas afetam a si mesmo e aos outros. Para contribuir com esta abordagem, apresentamos os resultados de uma pesquisa realizada a partir de um quadro interdisciplinar que combina os modelos do Conhecimento Didático do Conteúdo (CDC), as contribuições da natureza da ciência e a interrogação sobre os aspectos éticos do ensino. Uma sequência didática foi implementada com um grupo de professores de ciências argentinas em um curso de pós-graduação utilizando um recurso audiovisual para a análise de uma aula de matemática. As respostas a um questionário foram avaliadas por meio da análise de conteúdo e da aplicação do teste de sensibilidade ética (REST). Os resultados mostraram a capacidade de os professores reconhecerem e refletirem sobre os aspectos pedagógicos das práticas educacionais, enquanto as questões éticas permaneceram em um nível mais superficial. A menor importância dada a questões éticas nos alerta para a necessidade da incorporação do conhecimento ético como um novo componente do $\operatorname{CDC}$ e, portanto, a formação explícita da faculdade nesses aspectos.

Palavras-chave

formação de professores; pesquisa pedagógica; material audiovisual; ética; responsabilidade do professor 


\section{Introducción}

Las ciencias experimentales y la tecnología se encuentran en permanente transformación, por eso su enseñanza requiere una actualización tanto en los aspectos disciplinares como en las formas de enseñanza; para ello, la formación en servicio de los profesores en sus propios contextos de actuación es una estrategia privilegiada (Zúñiga et ál., 2014), que además permite explicitar la estructura ética de la ciencia (Gutiérrez, 2016). A lo largo de nuestros diferentes dispositivos formativos para los profesores de ciencia hemos detectado algunas dificultades vinculadas con la toma de decisiones en relación con situaciones que involucran temas controversiales como el consumo de drogas, o los derechos ligados con la vida y la muerte, por mencionar algunos. Dada la relevancia de estos temas emergentes, han sido incluidos como un problema específico a ser investigado en el marco de nuestros proyectos de investigación.

En este trabajo presentamos los resultados de un estudio en donde se planteó el análisis de una clase especialmente diseñada para un grupo de profesores de ciencias que se encontraba realizando una formación de posgrado en la universidad. El diseño incluyó la proyección de un recurso audiovisual (Brabeck et ál., 2000) correspondiente a un cortometraje denominado Clase de matemáticas en el contexto de una secuencia didáctica; de este modo, se pudo explicitar concepciones de los profesores sobre diferentes aspectos de la enseñanza, el aprendizaje y especialmente las cuestiones éticas relativas a estos procesos. A partir de la revisión crítica de la clase de "otro" en un momento posterior, los docentes pudieron reflexionar sobre la propia práctica y evidenciar sus aciertos, así como explicitar nuevas demandas de formación.

\section{Antecedentes}

La importancia de la capacitación de profesores en servicio es un tema abordado desde distintas orientaciones, tanto teóricas como metodológicas, con variaciones que incluyen el lugar y contexto de la investigación, así como el nivel educativo al cual se hace referencia (Caena, 2011; Darling, 2006; Escudero, 2006; 2011 ; Timperley et ál. 2008; Wei et ál., 2010; Viennot et ál., 2018). Una capacitación no solo guarda relación con los contenidos disciplinares y pedagógicos, sino también con la posibilidad de mantener una práctica reflexiva y crítica de la construcción del conocimiento científico y su transferencia a las prácticas educativas (Caballero et ál., 2015). Sin embargo, muchas de estas propuestas se presentan de manera superficial - fragmentada, dividiendo la teoría de la práctica, desvinculadas de las necesidades de los profesores y de los estudiantes, o bien fundamentadas en conceptos de déficit y no de crecimiento (Ávalos, 2002; Hawley et ál., 1998). Consecuentemente, el desarrollo profesional de los profesores resulta una actividad muy compleja que requiere de la integración de instancias metacognitivas que atiendan tanto a los aspectos personales, disciplinares, contextuales como a la propia práctica entre otros posibles (Vázquez et ál., 2007).

\section{Marco teórico o conceptual}

Desde la complejidad y multidimensionalidad que implica la realidad del aula, para esta investigación se plantea un marco teórico que combina los aportes de diferentes programas de investigación (Figura 1), tomando como eje el modelo del Conocimiento Didáctico del Contenido (CDC) (Shulman, 1986) al que concurren los aportes de la Naturaleza de la Ciencia (NOS) (Acevedo et ál., 2005) y el enfoque Ciencia 
Tecnología Sociedad (CTS) (Membiela, 1997), como soporte para el estudio de las cuestiones éticas particularmente vinculadas con la enseñanza de las ciencias.

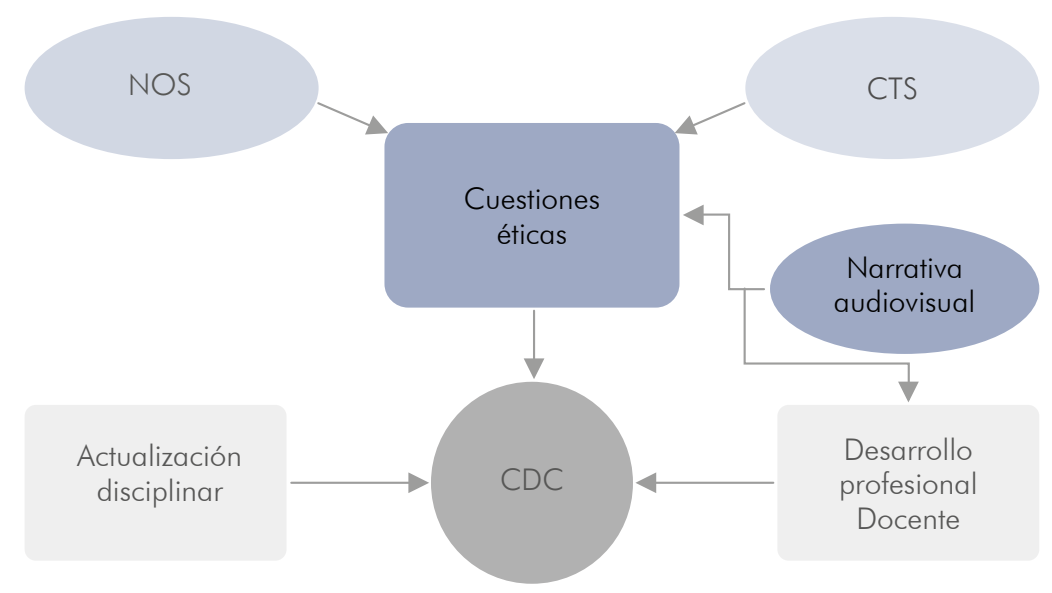

Figura 1. Convergencia de modelos teóricos

Fuente: elaboración propia.

Para poder enseñar su asignatura, el profesor recurre a un conocimiento complejo que ha ido construyendo a lo largo de su vida, a partir de su experiencia escolar y su trayectoria académica. En este conocimiento, O CDC, se integra la formación disciplinar con conocimientos de otra naturaleza (históricos, epistemológicos, sociales, contextuales, curriculares, institucionales y pedagógicos) (Garritz et ál., 2014), el cual permite a los profesores transponer el conocimiento disciplinar en un conocimiento enseñable, a fin de que pueda ser aprendido por sus estudiantes, eligiendo los mejores ejemplos, analogías, demostraciones y otras estrategias didácticas para un determinado grupo de alumnos. Si bien, el modelo del CDC ha comenzado a permear otras áreas de conocimiento como las clases de filosofía (Cruz et ál., 2020), o para la enseñanza de la historia (Tuithof et ál., 2019), es para el área de las ciencias de la naturaleza donde ha tenido su mayor desarrollo.

Desde su surgimiento, el modelo del CDC ha sido objeto de diversas conceptualizaciones (pueden consultarse, por ejemplo: Abell, 2008; Demirdöğen et ál., 2016; Loughran et ál., 2008). En las revisiones más recientes, ha habido una amplia discusión sobre cuáles serían los dominios o componentes que constituyen este tipo particular de conocimiento y hasta se han establecidos diferentes niveles, tales como el CDC colectivo, personal y el CDC en uso que se manifiesta en la acción (Carlson et ál., 2019; Gess-Newsome, 2015; Rodríguez et ál., 2019). Sin embargo, no ha sido considerado hasta hoy un conocimiento que atraviesa la totalidad de la práctica docente y que resulta imprescindible para la enseñanza esto es: el conocimiento ético. Estas interrelaciones han mostrado un área de vacancia para la investigación en didáctica de las ciencias, por lo que hemos iniciado una nueva línea de investigación para indagar las posibles articulaciones 
entre las cuestiones propias de las ciencias experimentales, los aspectos de naturaleza de la ciencia (Faikhamta, 2013; Lederman et ál., 2012) y los enfoques CTs (Lorenzo et ál., 2016; Ruiz et ál., 2013; Solbes et ál., 1996; 2001), y los posibles cuestionamientos vinculados con aspectos éticos que atraviesan las situaciones de enseñanza y de construcción del conocimiento científico (Núñez, 2004).

Las cuestiones éticas pueden considerarse como parte de los contenidos sociales desde el enfoque CTS (Garritz et ál., 2015), que son enriquecidos y a su vez enriquecen a las disciplinas metacientíficas como la historia, la filosofía o la epistemología de las ciencias desde las nuevas propuestas didácticas que incluyen los contenidos de la naturaleza de la ciencia (NOS) a la enseñanza de la ciencia y la tecnología (Adúriz-Bravo, 2011). Entre las diversas cuestiones éticas que conforman el conocimiento docente encontramos valores, actitudes y estilos de comportamiento (García et ál., 2009) y, evidentemente, la responsabilidad sobre estas (Gutiérrez, 2016).

Asimismo, se ha señalado que el CDC de los docentes de ciencias experimentales en el área de cuestiones éticas necesita ser mejorado y enriquecido para una transacción efectiva en el aula, así como para generar una auténtica comprensión ética entre los alumnos (Saxena, 2019)

Según el modelo de Rest (1979) el comportamiento ético es un fenómeno multideterminado y uno de sus componentes es la sensibilidad ética, que se centra en las acciones valorativas y en cómo cada acción afecta a sí mismo y a los otros. Por otra parte, la ética plantea la reflexión y valoración de normas y valores instituidos y repetidos por una cultura de acuerdo con principios universalizables en función de la condición humana, a diferencia de la moral que refiere a una época y a una cultura social determinada (Michel, 2000). Así, la ética proporciona razones que justifican o no las acciones, analizando los comportamientos y sus razones (Bolívar, 2005).

Por esta vía de argumentación, el cine, las series de televisión y otros formatos audiovisuales ponen en acto situaciones dilemáticas y controversiales que pueden servir de insumo para el diseño de diferentes propuestas de enseñanza. Las imágenes y las historias relatadas, si bien son ficcionales, resultan estimulantes para estudiantes y docentes, mientras amplían su conocimiento conceptual y fomentan sus capacidades críticas (Dark, 2005; García, 2011 ; Grilli, 2016; Petit et ál., 2015; 2016; Sierra, 2007). Así, el formato audiovisual permite una indagación reflexiva sobre cuestiones educativas, aplicable al estudio de las situaciones de enseñanza y aprendizaje de las ciencias (Perales, 2005; Petit et ál., 2012; Pro-Bueno et ál., 2005), siendo un recurso invaluable en la formación de profesores.

\section{Metodología}

Se empleó la narrativa audiovisual como estrategia para la explicitación y análisis de las concepciones de los profesores sobre cuestiones éticamente controvertidas (Cambra, 2016); se propuso una investigación educativa críticamente comprometida para la transformación de las prácticas convencionales a la vez que genere nuevo conocimiento (Murillo et ál., 2017); y se tomó como base una secuencia didáctica para profesores de escuela secundaria, especialmente diseñada para la detección de situaciones éticamente controversiales con base en la serie Breaking Bad (Cambra et ál., 2018). A partir de una secuencia cinematográfica, implementamos un taller destinado a docentes de ciencias experimentales en el cual se recoge información con respecto a la sensibilidad ética. 


\section{Contexto}

En el marco de una formación de nivel superior (UNC, s.f.) realizada con el fin de potenciar las relaciones entre el saber científico y tecnológico con su enseñanza e impactar positivamente en el desarrollo de las competencias docentes (Perrenoud, 2004; Pozo et ál., 2007; Tejeda Fernández, 2009; Valcárcel, 2005), implementamos el curso Fundamentos para el aprendizaje de la ciencia y la tecnología, en el año 2017. Con el propósito de llevarlo a cabo, se eligió un modelo que promueve la conformación de comunidades de aprendizaje docente (Liesa et ál., 2018), a fin de enfrentar con sentido crítico los retos que generan las tendencias del desarrollo científico y tecnológico.

A lo largo del curso se abordaron diferentes cuestiones características de las prácticas educativas con el fin de comprender los procesos y las circunstancias que intervienen en el aprendizaje de las ciencias experimentales y la tecnología en diferentes niveles del sistema educativo. Se parte de la base de que todos los alumnos del curso ejercen o han ejercido la actividad docente en alguna de las disciplinas que componen el área: química, física, ciencias biológicas u otras relacionadas a estas. La propuesta general del curso procura responder a preguntas tales como ¿̇Por qué este tema es tan difícil de entender para mis estudiantes?, ¿̇por qué esta actividad resulta tan interesante y motivadora para estos estudiantes, pero no lo es para aquellos? ¿̇podría probar una nueva forma de enseñar este contenido?, ¿̇cuáles son los aspectos éticos involucrados con los distintos contenidos? y żqué aportes han hecho la Psicología del Aprendizaje y la Didáctica de las Ciencias a esta problemática? En definitiva, se intenta promover la profesionalización académica a través de conocimientos teóricos y metodológicos para estimular la revisión de las prácticas educativas.

\section{Participantes}

Participaron voluntariamente de este estudio diecinueve profesores del área de las ciencias experimentales, mayoritariamente mujeres, que se encontraban realizando el mencionado curso. A los fines de esta investigación no se consideró relevante diferenciar los perfiles individuales de los participantes y todos expresaron su consentimiento de participar en la investigación y sus respuestas fueron relevadas anónimamente.

\section{Instrumento}

Se utilizó el REST (Racial and Ethical Sensitivity Test) concebido como una herramienta audiovisual de investigación y diagnóstico psicológico para evaluar el grado de sensibilidad ética entre los educadores (Brabeck et ál., 2000; Michel et ál., 2008; Sirin et ál., 1999; Rest, 1983; Rogers-Sirin et ál., 2010). El REST fue desarrollado por Mary Brabeck en Estados Unidos en el año 2000, traducido, subtitulado y 
adaptado en el Programa de Investigación de la Universidad de Buenos Aires desde 2003 (Michel, et ál., 2008).

Se seleccionó el vídeo Clase de matemáticas, que en sus cuatro minutos de duración presenta una clase del profesor Ross, quien tiene larga trayectoria en la enseñanza de matemática avanzada y debe hacerse cargo de un curso introductorio de matemática básica. El vídeo expone una situación de clase del profesor frente a sus alumnos en presencia de la directora del departamento de matemática; así, la secuencia muestra distintas cuestiones sobre los procesos comunicativos, la integridad, el hostigamiento, la diversidad cultural, la discriminación social y de género. Las cuestiones éticas incluidas en el vídeo se indican en la Tabla 1.

Tabla 1. Características de la herramienta REST

\begin{tabular}{|c|c|c|}
\hline Ítem & Escena incluida en el vídeo & Componente de sensibilidad ética \\
\hline 1 & Falta de autoevaluación del profesor Ross & Integridad, competencia \\
\hline 2 & Falta de información del profesor Ross sobre la enseñanza & Competencia, integridad \\
\hline 3 & Falta de respeto del profesor Ross por sus alumnos & $\begin{array}{l}\text { Respeto por los derechos y la dignidad de los demás, } \\
\text { responsabilidad profesional }\end{array}$ \\
\hline 4 & $\begin{array}{l}\text { Tratamiento diferencial del profesor Ross para los } \\
\text { estudiantes basado en la raza }\end{array}$ & $\begin{array}{l}\text { Integridad, respeto por los derechos y la dignidad de los } \\
\text { demás }\end{array}$ \\
\hline 5 & Trato diferencial del profesor Ross basado en el género & Integridad, respeto por los derechos y la dignidad de los demás \\
\hline 6 & $\begin{array}{l}\text { La responsabilidad de la profesor Cruz de confrontar al } \\
\text { profesor Ross }\end{array}$ & $\begin{array}{l}\text { Responsabilidad profesional, preocupación por el bienestar } \\
\text { de los demás }\end{array}$ \\
\hline
\end{tabular}

Fuente: elaboración propia.

Diseño, implementación de la secuencia y procedimiento de recolección de datos

La muestra quedó conformada por el corpus de respuestas escritas una vez finalizada la implementación de la actividad. En un primer momento se presentó a los participantes el video incrustado en una presentación de Power Point, en dos veces consecutivas, para brindar la oportunidad a los participantes de detectar las situaciones expuestas. Seguidamente, se aplicó el cuestionario escrito (Figura 2) a ser respondido de manera individual, de a una pregunta por vez. Las respuestas a cada una de las preguntas fueron recogidas por los investigadores en el orden que se formularon.

1) El profesor Ross finaliza diciendo: "esta no es una clase de matemática avanzada. Pero pienso que estoy haciendo progresos con ellos żno lo cree?"

Ahora imagine que Ud. es Marisa Cruz, la directora del departamento de matemática que observa la clase. ¿̇ué le respondería al profesor Ross?

2) ¿Cómo piensa usted que reaccionaría el profesor Ross ante lo que usted le dice?

3) ¿Cómo cree usted que los estudiantes reaccionarían ante su comentario al profesor Ross?

4) ¿Qué situaciones o hechos que aparecen en la escena le parece a usted que son cuestionables para un docente?

Figura 2. Cuestionario individual escrito

Fuente: elaboración propia. 
Por último, se planteó un debate en plenario para la discusión y el intercambio de opiniones de las respuestas siguiendo las preguntas enunciadas en la Figura 3 en esta instancia se promovió el análisis colectivo de la práctica educativa del profesor Ross en relación con los contenidos de todo el curso y haciendo reflexión sobre la propia práctica.

" ¿ंQué aspectos del video les llamaron más la atención?

» ¿̇ué elementos estaban presentes? ¿̇podemos relacionarlos con algo que ya hayamos visto (en el desarrollo del curso)?

¿ ¿Qué importancia se le asigna en el video al tema de la clase?

Figura 3. Análisis de las prácticas educativas

Fuente: elaboración propia.

\section{Análisis de los datos}

Los textos de las respuestas escritas fueron analizados desde una perspectiva cuali-cuantitativa empleando dos estrategias de modo complementario:

a. Análisis de la totalidad de los textos escritos. Clasificación de los elementos constitutivos de las líneas argumentales establecidas en sus respuestas mediante análisis de contenido (Bardin, 1996) y validación inter-pares para garantizar su fiabilidad.

b. Para la pregunta 4 del cuestionario se aplicó la categorización de la herramienta REST a fin de describir los aspectos generales de la sensibilización ética, en los que pueden describirse tres niveles de complejidad según se resume en la Tabla 2.

Tabla 2. Niveles de sensibilidad ética

\begin{tabular}{cl}
\hline Nivel de complejidad & \multicolumn{1}{c}{ Descripción } \\
\hline 1 & No detecta \\
\hline 2 & Solo detecta (reconocimiento de un aspecto) \\
\hline 3 & Reconoce complejidad de la situación (más de un aspecto) \\
\hline
\end{tabular}

Fuente: elaboración propia.

\section{Resultados y discusión}

El análisis de las respuestas escritas permitió detectar cuatro aspectos esenciales de las prácticas educativas a los cuales los participantes prestaron especial atención a partir de la exposición del vídeo (previamente al debate en plenario). En primer lugar, surgió el concepto de clima de clase y cuestiones afectivas como un tema que preocupación de los docentes. En seguida, aspectos vinculados con las estrategias de enseñanza; seguido por aspectos particulares del conocimiento 
didáctico del contenido, y para finalizar las cuestiones éticas. Estas categorías, surgidas ad hoc a partir del análisis de los datos recogidos, ponen de manifiesto los temas en los cuales los profesores expresan sus inquietudes haciendo visibles aquellos en los cuales su interés es menor o no se expresa. A continuación, se discuten los resultados y se incluyen a modo de ejemplo algunas frases literales extraídas de las respuestas de los participantes.

\section{Clima de clase. Relación docente-alumno}

Las cuestiones relacionadas al trato con los alumnos y la situación en el aula fueron planteadas como un aspecto muy importante; así, en sus respuestas, los docentes señalaron que el profesor de matemáticas desconoce el mal clima de trabajo que genera (y su equivocación al creer que está haciendo progresos con los estudiantes), ya que tiene conductas que no son adecuadas, lo cual genera incomodidad en sus alumnos.

También mencionaron como aspectos negativos que el profesor Ross no conoce el nombre de sus estudiantes ni tampoco escucha sus inquietudes, llegando a confrontar abiertamente con ellos. Algunas respuestas aludieron a que el profesor no tenía autocrítica, puesto que trata de irrespetuosos a los alumnos cuando es él quien comienza faltando el respeto, además de exigirles conocimientos, aunque él no los había enseñado.

No se da cuenta que sus alumnos no entienden y además supone que está realizando grandes avances. (Respuesta de participante)

Además, en las respuestas se evidenció el reconocimiento de una fuerte asimetría en la relación docente-alumno, en donde el profesor es quien detenta un saber absoluto dejando a sus alumnos en un lugar pasivo de obediencia, desde el cual no los motiva a aprender ni a participar. Completando el cuadro, señalaron que el exceso de protagonismo del profesor (ya que intenta demostrar continuamente su saber) va en detrimento de la posibilidad de enseñar realmente.

La distancia del docente como centro de acumulación de conocimiento y el alumno en situación pasiva.

Ser un poco más humano con sus alumnos. Creo que bajándose del pedestal en que lo pusieron o solo se subió, vería sus múltiples errores. (Respuesta de participante)

Tener la voluntad de querer enseñar y no solo demostrar que él lo sabe todo

En definitiva, los docentes participantes pudieron reconocer en las prácticas educativas de otro (el profesor Ross) ciertos comportamientos comprometidos con la ética, en relación con una buena enseñanza vinculados a la relación entre el profesor y sus estudiantes.

\section{Estrategias de enseñanza}

El análisis de las respuestas puso en evidencia tanto el reconocimiento de estrategias de enseñanza como la valoración de los modos en que dichas estrategias eran utilizadas en el aula por el profesor Ross. Reconocieron a la exposición como la principal estrategia de enseñanza, en la que detectaron además algunos defectos tales como hablar mientras se mira a la pizarra de espaldas a la clase, ignorar los conocimientos previos de los estudiantes e utilizar un lenguaje técnico desconocido para ellos e ignorar sus preguntas.

Adicionalmente, señalaron que Ross parecía poseer los conocimientos necesarios como profesor de matemáticas, pero que no sabía cómo enseñarlos o que su modo de enseñar no resultaba eficaz porque los recursos 
didácticos que utilizaba no eran apropiados, ni la secuencia de contenidos (no explica el tema nuevo y pasa directamente a la ejercitación), ni la dinámica de la clase (simulación dialógica).

Reformulación del problema con las mismas estrategias que no permitieron comprender al mismo en una instancia previa.

Saltar la explicación previa y comenzar a resolver situaciones problemáticas, que terminan siendo descontextualizadas para los alumnos y pueden generar frustraciones. (Respuesta de participante)

Los participantes consideraron que el docente debería haber respondido las dudas o consultas de los alumnos cuando ellos le decían que no entendían, también dieron importancia a conocer a los estudiantes (por su nombre, sus características e intereses), ya que de esta manera se redundaría en una mayor integración del grupo y propiciaría el aprendizaje. Otro aspecto mencionado como negativo fue que el profesor presuponía que sus alumnos no entendían, como un problema relacionado con sus características personales sin tener en cuenta la calidad de su propia explicación y su actitud frente a la clase.

Cabe señalar que los docentes participantes concentraron su atención en las deficiencias de la clase, e ignoraron en sus comentarios cuestiones como la utilización de libro de texto o la elaboración de un material didáctico, que Ross repartió a sus estudiantes; lo cual hubiera ampliado el análisis global de esta práctica educativa en particular.

\section{Conocimiento didáctico del contenido}

Algunas respuestas mostraron ideas vinculadas con la importancia del CDC; por ejemplo, reconocieron al conocimiento disciplinar (en este caso, las matemáticas) como un componente central, aunque disociado de los conocimientos pedagógicos necesarios para su explicación durante la enseñanza. Estrictamente en relación con los contenidos, las respuestas señalaron que la explicación no era pertinente con el tema, dado que lo presenta de forma compleja, y que aun así el docente pretendía que los alumnos respondieran satisfactoriamente; es decir, que de manera indirecta los docentes participantes reconocieron la necesidad de poseer un buen CDC para poder desempeñar con éxito la tarea de enseñanza.

No realiza un análisis didáctico de los contenidos y por el modelo expuesto, da la impresión de que no se traspuso el contenido.

Me parece que el profesor Ross no tiene técnica de enseñanza, podrá saber mucho del tema pero si no lo puede transmitir no es válido. (Respuesta de participante)

En las declaraciones elegidas como ejemplo, se pueden reconocer diferentes niveles de uso del vocabulario técnico didáctico. En el primero de ellos, aparecen términos como "análisis didáctico", "modelo" y una referencia explícita 
a la transposición didáctica, los que resultan en un indicador de un mayor conocimiento didáctico que el del segundo ejemplo, en el cual aparecen expresiones más vinculadas al lenguaje cotidiano "técnica de enseñanza" y hasta errores conceptuales como el referirse a la "transmisión" del conocimiento. No obstante estas diferencias, en ambos casos se plasmó la reflexión sobre la importancia del CDC.

\section{Cuestiones éticas de la}

enseñanza (sensibilidad

\section{para reconocer e interpretar situaciones dilemáticas)}

Con relación a las cuestiones éticas de la enseñanza, los docentes mencionaron actitudes discriminatorias por parte del profesor hacia algunos de sus estudiantes. Estas fueron reconocidas en sus bromas (de mal gusto) y en los ejemplos que utilizaba como parte de la explicación; también señalaron la falta de respeto hacia el alumnado y la presencia de ciertos prejuicios del profesor debidos a la condición sociocultural de los estudiantes, los cuales fueron considerados como hostigamiento. Otro aspecto mencionado en algunas respuestas fue la tendencia del profesor Ross a discriminar por género, ya que su trato con sus alumnas es aún peor que con sus estudiantes varones.

Dos de las respuestas aportadas plantearon la reflexión en otra dirección apelando a los motivos que explicarían las razones por las cuales el profesor Ross considera que ha hecho progreso con los alumnos, las cuales fueron planteadas como preguntas:

Estimado profesor, Żen base a qué parámetros usted dice que está haciendo progresos con ellos? Si usted estuviera en lugar de ellos, ટ̇creería que el profesor le ha dado los elementos como para entender y "progresar" en la temática?", ¿̇por qué piensa usted que hay un avance?, ¿̇cómo han sido sus clases anteriores?

(Respuesta de participante)

Particularmente, para el análisis de la pregunta 4 "¿̇Qué situaciones o hechos que aparecen en la escena le parece a usted que son cuestionables para un docente?", se aplicó la puntuación establecida por el instrumento REST según los valores que se indican en la Tabla 3.

Tabla 3. Puntuaciones de la pregunta 4 aplicando el instrumento REST

\begin{tabular}{|c|c|c|c|c|}
\hline Ítem & Descripción & 1 & 2 & 3 \\
\hline 1 & $\begin{array}{l}\text { Falta de conciencia y autoevaluación del profesor Ross sobre los propios valores y } \\
\text { prejuicios (integridad, competencia) }\end{array}$ & 0.15 & 0.79 & 0.05 \\
\hline 2 & Falta de conocimiento pertinente para la enseñanza (competencia, integridad) & 0 & 0.95 & 0.05 \\
\hline 3 & $\begin{array}{l}\text { Falta de respeto hacia sus alumnos (respeto por los derechos y la dignidad de los } \\
\text { demás, responsabilidad profesional y social) }\end{array}$ & 0.10 & 0.84 & 0.05 \\
\hline 4 & $\begin{array}{l}\text { Trato diferencial hacia sus alumnos basado en su origen racial (integridad, } \\
\text { discriminación, responsabilidad profesional y social) }\end{array}$ & 0.89 & 0.10 & 0 \\
\hline 5 & $\begin{array}{l}\text { Trato diferencial hacia los alumnos basado en el género (integridad, } \\
\text { responsabilidad profesional y social, sexismo) }\end{array}$ & 0.95 & 0.05 & 0 \\
\hline 6 & $\begin{array}{l}\text { Responsabilidad de la profesor Cruz de confrontar al profesor Ross } \\
\text { (responsabilidad profesional, preocupación por el bienestar de los alumnos) }\end{array}$ & 1.00 & 0 & 0 \\
\hline
\end{tabular}


En principio, se observó que en los primeros tres ítems propuestos (ítems 1, 2 y 3) la mayoría de los docentes encuestados reconoció parcialmente los elementos problemáticos presentados en la situación ficcional. En general, los participantes abordaron las cuestiones sin profundizar sobre ellas sin hacer referencia ni sugerir un curso de acción posible con el fin de remediar el aspecto considerado como negativo. Solamente en una de las respuestas pudo evidenciarse un mayor despliegue y complejidad para cada una de las categorías analizadas. De manera complementaria, para el segundo grupo de ítems $(4,5$ y 6$)$ no se detectaron los elementos éticos incluidos en el instrumento en las respuestas.

En la Figura 4 puede apreciarse que la mayoría de los participantes consideraron como elementos cuestionables aquellos vinculados a los ítems 1 (falta de conciencia sobre sus valores y prejuicios) y 2 (falta de conocimiento-contenido pertinente para la enseñanza), que se vinculan a la competencia e integridad del docente. Del mismo modo, en el ítem 3, se reconoció que el profesor Ross no respetaba a sus alumnos, lo cual se encuentra ligado a la responsabilidad profesional y social. Dicha categoría también se encuentra presente en los ítems 4,5 y 6 , pero no fue reconocida referida al trato diferencial del profesor hacia sus alumnos, basado en su origen racial o género. En cuanto a la responsabilidad de la profesora Cruz de confrontar al profesor Ross tampoco fue tenida en cuenta, esto podría deberse a que los últimos tres ítems del cuestionario poseen mayores niveles de abstracción, por lo cual requerirían cierto entrenamiento para lograr la reflexión en relación con el dilema ético en juego.

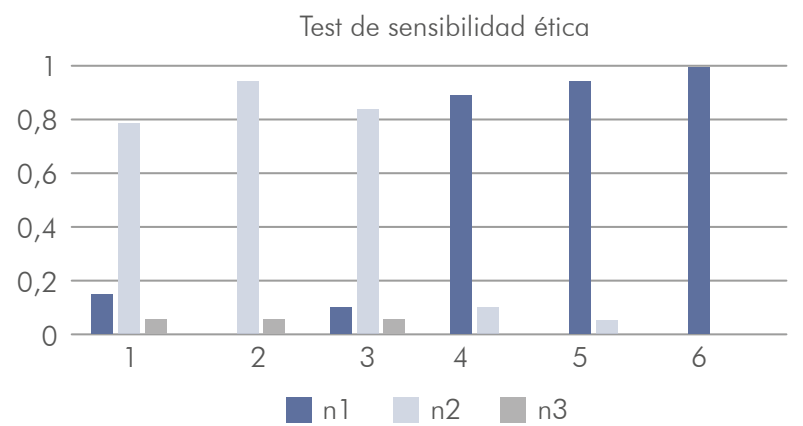

Figura 4. Niveles de sensibilidad ética para los ítems considerados Fuente: elaboración propia.

En síntesis, desde el punto de vista de la sensibilidad ética, es posible categorizar las respuestas de la siguiente manera:

- Subestimación a los estudiantes: el docente tiene el preconcepto de que los alumnos no entienden y no poseen los conocimientos adecuados. Los culpa del fracaso de la clase sin revisar su metodología pedagógica.

Considerar que los alumnos no saben. El profesor Ross es un sujeto que subestima a sus estudiantes, no valora sus aportes. Cuando hacen una pregunta se los toma de inútiles. 
- Intolerancia y autoritarismo: el docente adopta una posición de superioridad ante los estudiantes y no los trata con respeto.

No debe amenazarlos con castigarlos.

No dar la posibilidad de diálogo a los alumnos para la expresión de pareceres que puedan favorecer la relación docente-alumno.

- Discriminación: el profesor realiza juicios de valor sobre los alumnos, hace chistes sobre sus vidas personales basándose en su origen social. También señalan que hace diferencia en cuanto al género, ya que da la prioridad a un estudiante hombre y no a una mujer, evidenciando ciertas preferencias.

Utilizar irónicamente situaciones cotidianas que apelan a una imagen social negativa cargadas a sus alumnos.

Menospreciar las capacidades cognitivas de sus alumnos por su origen social.

Marcar las diferencias sociales, de clases sociales, entre alumnos, y remarcar lo negativo de dicha clase social (ejemplo negro o latino, ladrón; blanco, bueno).

Si focalizamos en las dimensiones éticas que el material presenta, las respuestas de los encuestados estarían asociando la falta de conciencia sobre los valores y prejuicios del profesor Ross (ítem 1) con el trato del docente hacia los alumnos y con el clima de trabajo generado en el aula. Sin embargo, en ninguna de las respuestas se hizo evidente que ello esté vinculado a la integridad del docente, ni se señaló que la falta de consciencia del profesor Ross sobre sus propios prejuicios requiera ser abordada, teniendo en cuenta la complejidad de la cuestión. Tampoco relacionaron este hecho con cierta deficiencia en las competencias pedagógicas del docente.
Más aun, la gran mayoría de las respuestas declaró que el contenido no resultaba pertinente para la enseñanza en un curso de matemática básica (ítem 2), lo cual plantearía cierta falencia en el proceso de enseñanza. En este sentido, dieron cuenta de la falta de responsabilidad del docente para la correcta preparación de su clase considerando los conocimientos previos y los recursos adecuados para suplementar su explicación. Muchas de las respuestas enfatizaron la disyunción entre el conocimiento que el profesor Ross tiene de los contenidos y la forma en que los comunica a su alumnado, ligándolo más a cuestiones pedagógicas que éticas.

Además, una amplia mayoría de los participantes aludió a la falta de respeto del profesor Ross hacia sus alumnos (ítem 3), enfatizando el rol del docente y el modo en que ello repercute en la dinámica de la clase puesto que genera una posición pasiva en el alumno. Sin embargo, no reconocieron en sus respuestas el trato diferencial hacia los alumnos debido a su origen racial. Solamente dos de las respuestas explicitaron que se trataba de una conducta discriminatoria, remitiéndola a la utilización de malos ejemplos y bromas inapropiadas. En cuanto a la cuestión de género, solo una respuesta declaró que el docente "da la prioridad a un estudiante hombre y no una mujer", pero no profundizó sobre ello.

Un aspecto por destacar fue la ausencia de comentarios referidos a la responsabilidad de la profesora Cruz con respecto al desempeño del profesor Ross. El instrumento audiovisual muestra que ella presenció situaciones donde el profesor Ross denigraba a sus estudiantes y la falta de eficacia de sus métodos de enseñanza, pero no incluye su reacción para dar lugar a posibles interpretaciones por parte de los participantes. Siendo la jefa del departamento sería esperable que ella fuera capaz de confrontar al profesor Ross con el fin de
TECNÉ EPISTEME DIDAXIS 
promover el bienestar de los alumnos y llevar adelante acciones de cambio en las condiciones de trabajo en el aula, así como también abordar junto al profesor Ross sus prejuicios morales y poder trabajarlos. Sin embargo, ninguno de los docentes participantes en esta investigación realizó alguna reflexión al respecto.

En resumen, los resultados mostraron que, si bien fueron detectados la mayoría de los elementos problemáticos presentados en la situación, los docentes no lograron interpretar en profundidad ni reflexionar acerca de las cuestiones vinculadas con la ética (principalmente, los componentes de integridad, competencia y respeto por los derechos y la dignidad de los demás). A su vez, se observó que la sensibilidad a las cuestiones éticas se encuentra asociada a lo pedagógico en tanto algunas de las categorías propuestas por el REST involucran explícitamente las competencias del docente. El CDC quedó representado con algunas referencias puntuales a ciertos contenidos disciplinares, vistos de manera independiente a las estrategias utilizadas para su enseñanza, desvinculado las controversias éticas y de los aspectos emocionales y afectivos que concurren en las prácticas educativas.

\section{Conclusiones y perspectivas}

Los profesores participantes de esta investigación centraron su atención en el análisis de las dimensiones pedagógico-didácticas presentadas en el material audiovisual pero no lograron detectar cuestiones conectadas con los dilemas éticos presentes ex professo en el material. Estos resultados ponen de manifiesto la necesidad de una formación ética que contribuya a la conformación de un CDC más rico y complejo para hacer frente a las variadas situaciones de la enseñanza actual atravesada por las múltiples dimensiones de la diversidad. A los componentes clásicos del CDC en su momento se le incorporó la afectividad y las emociones (Mellado et ál., 2014), y ahora se hace evidente la incorporación de las cuestiones éticas, ya que el aprendizaje de las ciencias experimentales y la tecnología se encuentran íntimamente imbricadas con las cuestiones éticas de la sociedad en que vivimos. Dado que, la mayoría de los elementos problemáticos (situaciones dilemáticas) presentados en el recurso audiovisual fueron reconocidos de manera superficial se hace necesaria una revisión de las estrategias y políticas educativas pensadas para la formación inicial y en servicio de los profesores que contemplen la sensibilidad ética como un componente sustantivo en la constitución de su conocimiento didáctico, vinculado directamente con el campo disciplinar del desempeño docente.

De manera complementaria, esto implica necesariamente la necesidad de ampliar las investigaciones en esta nueva zona de interdisciplinariedad, para lo cual el uso de las narrativas audiovisuales con la combinación de la herramienta REST y el análisis de contenido vienen demostrando su versatilidad para el estudio de las concepciones y otras conceptualizaciones de profesores a partir del análisis crítico de la clase de "otro". Al poner inicialmente la mirada en el afuera, se puede 
avanzar en la reflexión llegando a la revisión de la propia práctica y poder así contribuir a la conformación del propio conocimiento profesional docente.

\section{Referencias}

Abell, S. (2008). Twenty Years Later: Does Pedagogical Content Knowledge Remain a Useful Idea? International Journal of Science Education, 30(10), 1405-1416. https://doi. org/10.1080/09500690802187041

Acevedo, J., Vázquez, A., Martín, M., Oliva, J., Acevedo, P., Paixão, M. y Manassero, M. (2005). Naturaleza de la Ciencia y Educación Científica para la Participación Ciudadana. Una Revisión Crítica. Revista Eureka sobre Enseñanza y Divulgación de las Ciencias, 2(2), 121-140. https://doi. org/10.25267/Rev_Eureka_ensen_divulg_ cienc.2005.v2.i2.01

Adúriz-Bravo, A. (2011). Desde la enseñanza de los "productos de la ciencia" hacia la enseñanza de los "procesos de la ciencia" en la Universidad. Cuadernillos de actualización para pensar la Enseñanza Universitaria, 6(3), 5-12.

Ávalos, B. (2002). Profesores para Chile: historia de un proyecto. Ministerio de Educación.

Bardin, L. (1996). Análisis de contenido. Akal.

Bolívar, A. (2005). El lugar de la ética profesional en la formación universitaria. Revista Mexicana de Investigación Educativa, 10(24), 93-123.

Brabeck, M., Rogers, L., Sirin, S., Henderson, J., Benvenuto, M. y Weaver, M. (2000). Increasing Ethical Sensitivity to Racial and Gender Intolerante in Schools: Development of the Racial Ethical Sensitivity Test. Ethics y Behavior 10(2), 119-137. https://doi.org/10.1207/ S15327019EB1002_02

Caballero, K. y Bolívar, A. (2015). El profesorado universitario como docente: hacia una identidad profesional que integre docencia e investigación. REDU. Revista de Docencia Universitaria, 13(1), 57-77. https://doi. org/10.4995/redu.2015.6446

Caena, F. (2011). Literature Review Quality in Teachers' Continuing Professional Development. Education and Training 2020. Thematic Working Group 'Professional Development of Teachers'. European Commission. http://ec.europa.eu/education/ policy/strategic-framework/doc/teacherdevelopment_en.pdf

Cambra, I. (2016). Psicología, bioética y narrativa cinematográfica: un análisis cualitativo de producciones de estudiantes sobre conflictos bioéticos relacionados con la identidad. Revista Latinoamericana de Bioética, 16(31-2), 16-39. https://doi. org/10.18359/rlbi. 1464

Cambra, I. y Lorenzo, M. (2018). Entrelazando la ética con las ciencias experimentales: una propuesta didáctica para la capacitación de profesores con la serie Breaking Bad. Didáctica de las Ciencias Experimentales y Sociales, 34, 105-122. https://doi.org/10.7203/ dces.34.11478

Carlson, J. y Daehler, K. (2019). The Refined Consensus Model of Pedagogical Content Knowledge in Science Education. En A. Hume, R. Cooper, A. Borowski A. (Eds.). Repositioning Pedagogical Content Knowledge in Teachers' Knowledge for Teaching Science, https://doi.org/10.1007/978-981 13-5898-2_2 EPISTEME 
Dark, M. L. (2005). Using Science Fiction Movies in Introductory Physics. The Teacher Physics, 43, 463-465. https://doi.org/10.1119/1.2060648

Darling-Hammond, L. (2006). Constructing 21 st-Century Teacher Education. Journal of Teacher Education, 57(3), 300-314. https://doi.org/10.1 177/0022487105285962

Demirdöğen, B., Hanuscin, D., Uzuntiryaki-Kondakci, E. y Köseoğlu F. (2016). Development and Nature of Preservice Chemistry Teachers' Pedagogical Content Knowledge for Nature of Science. Research in Science Education, 46(4), 575-612. https://doi.org/10.1007/s $11165-015-9472-z$

Escudero, J. M. (2006). La formación del profesorado y el derecho de una buena educación para todos. En J. Escudero y A. Luis (Coords.). Formación del Profesorado y Educación de calidad para todos (p. 17-50). Octaedro.

Escudero, J. M. (2011). La formación continuada del profesorado, un tema crucial para la mejorar la educación. Ministerio de Educación.

Faikhamta, C. (2013). The Development of in-Service Science Teachers' Understandings of And Orientations to Teaching the Nature of Science Within a PCKbased NOS course. Research in Science Education, 43, 847-869. https://doi. org/10.1007/s $11165-012-9283-4$

García, F. (2011). Las escenas cinematográficas: una herramienta para el estudio de las concepciones alternativas de física y química. Revista Eureka sobre enseñanza y divulgación de las ciencias, 8(3), 291-311.

García, R., Sales, A., Moliner, O. y Ferrández, R. (2009). La formación ética profesional desde la perspectiva del profesorado universitario. Teor. educ., 21 (1), 199-221. https://doi.org/10.25267/Rev_Eureka_ensen_divulg_cienc.2011.v8.i3.06

Garritz, A., Lorenzo, G. y Daza, S. (2014). Conocimiento didáctico del contenido. Una perspectiva iberoamericana. Editorial Académica Española. https://doi. org/10.1016/S0187-893X(15)72101-4

Gess-Newsome, J. (2015). A Model Of Teacher Professional Knowledge and Skill Including PCK: Results of the Thinking From the PCK Summit. En A. Berry, P. Friedrichsen y J. Loughran (Eds.). Re-Examining Pedagogical Content Knowledge in Science Education (pp. 28-42). Routledge.

Grilli, J. (2016). Cine de ciencia ficción y enseñanza de las ciencias. Dos escuelas paralelas que deben encontrarse en las aulas. Revista Eureka sobre enseñanza y divulgación de las ciencias, 13(1), 137-148. https://doi.org/10.25267/ Rev_Eureka_ensen_divulg_cienc.2016.v13.i1.10

Gutiérrez, C. (2016). Los valores de la actividad científica en la enseñanza de las ciencias desde la perspectiva sociocultural. Tecné, Episteme y Didaxis: TED. Número Extraordinario, 832-836.

Hawley, W., y Valli, L. (1998). The Essentials of Effective Professional Development. A New Consensus. En L. Darling-Hammond y G. Sykes (Eds.). Teaching as a 
Learning Professional. Handbook of Policy and Practice (pp. 127-149). Jossey-Bass.

Lederman, N. G., Antink, A., y Bartos, S. (2012). Nature of Science, Scientific Inquiry, and Socio-Scientific Issues Arising from Genetics: A Pathway to Developing a Scientifically Literate Citizenry. Science and Education, 23(2), 285-302. https://doi.org/10.1007/ s1 1191-012-9503-3

Liesa, E., Castelló, M. y Becerril, L. (2018). Nueva escuela, ¿̇nuevos aprendizajes? Revista de Estudios y Experiencias en Educación, Número Especial, 2, 15-29. https://doi.org/10.21703/rexe.Especial2_201815291

Lorenzo, M. G. y Farré, A. S. (2016). La ciencia y la tecnología entre el bien y el mal. Un debate para la formación ciudadana. Aesthethika, International Journal on Subjectivity, Politics and the Arts, 12(3), 35-42.

Loughran, J., Mulhall, P., Berry, A. (2008). Exploring Pedagogical Content Knowledge in Science Teacher Education. International Journal of Science Education, 30(10), 1301 - 1320. https://doi.org/10.1080/ 09500690802187009

Mellado, V., Borrachero, A., Brígido, M., Melo, L., Dávila, M., Cañada, F., Conde, M., Costillo, E., Cubero, J., Esteban, R., Martínez, G., Ruiz, C., Sánchez, J., Garritz, A., Mellado, L., Vázquez, B., Jiménez, R. y Bermejo, M. (2014). Las emociones en la enseñanza de las ciencias. Enseñanza de las Ciencias, 32(3), 11 -36. https://doi.org/10.5565/rev/ ensciencias. 1478

Membiela, P. (1997). Una revisión del movimiento educativo ciencia-tecnología-sociedad. Enseñanza de las Ciencias, 15(1), 51-57.

Michel, J. (2000). La ética en movimiento. Cuaderno de Humanidades, 1 (1) 15-25.
Michel, J., Salomone, G., Costa, G., Rodríguez, A., Luzzi, S. y Pérez, F. (2008). Sensibilidad ética en el ámbito educativo: la adaptación al español del Racial and Ethical Sensitivity Test (REST). Memorias del XV Jornadas de Investigación y Cuarto Encuentro de Investigadores en Psicología del Mercosur. Facultad de Psicología, Universidad de Buenos Aires.

Murillo, F. y Hidalgo, N. (2017). Hacia una investigación educativa socialmente comprometida. Revista Iberoamericana de Evaluación Educativa, 10(2), 5-8.

Núñez, J. (2004). Ética, ciencia y tecnología: sobre la función social de la tecnociencia. El saber ético de ayer a hoy. Editorial Félix Varela.

Perales-Palacios, F. y Vílchez-González, J. (2005). The Teaching of Physics and Cartoons: Can They be Interrelated In Secondary Education? International Journal of Science Education, 27(14), 1647-1670. https://doi.org/ $10.1080 / 09500690500206366$

Perrenoud, P. (2004). Diez nuevas competencias para enseñar. Graò.

Petit, M. F. y Solbes, J. (2012). La ciencia ficción y la enseñanza de las ciencias. Enseñanza de las Ciencias, 30(2), 55-72. https://doi. org/10.5565/rev/ec/v30n2.494

Petit, M. y Solbes, J. (2015). El cine de ciencia ficción en las clases de ciencias de enseñanza secundaria (I). Propuesta didáctica. Revista Eureka sobre enseñanza y divulgación de las ciencias, 12(2), 311-327. https://doi. org/10.25267/Rev_Eureka_ensen_divulg_ cienc.2015.v12.i2.06

Petit, M. y Solbes, J. (2016). El cine de ciencia ficción en las clases de ciencias de enseñanza secundaria (II). Análisis de películas. Revista Eureka sobre Enseñanza y Divulgación de 
las Ciencias, 13(1), 176-191.https://doi.org/10.25267/Rev_Eureka_ensen_divulg_cienc.2016.v13.i1.13

Pozo, J. y Monereo, C. (2007). Competencias para (con)vivir con el siglo xxl. Cuadernos de pedagogía, 370, 9-84.

Pro-Bueno, A. y Ezquerra-Martínez, A. (2005). ¿Qué ciencia ve nuestra sociedad? Alambique: Didáctica de las ciencias experimentales, 12(43), 37-48.

Rest, J. (1983). Morality. P. Mussen (Series Ed.). J. Flavell y E. Monkmam, E. (Eds.). Handbook of child psychology, 3, 556-629. Wiley. https://doi.org/10.1037/00220663.91 .4 .644

Rest, J., Narvaez, D., Thoma, S. y Bebeau, M. (1999). Devising and Testing a Revised Instrument of Moral Judgment. Journal of Educational Psychology, 91 (4), 644-659.

Rest, J.R. (1979). Development in Judging Moral Issues. University of Minnesota Press.

Rodriguez, J-M. y Towns, M. (2019). Alternative Use for the Refined Consensus Model of Pedagogical Content Knowledge: Suggestions for Contextualizing Chemistry Education Research. Journal of Chemical Education, 96(9), 1797-1083. https:// doi.org/10.1021/acs.jchemed.9b00415

Ruiz, J., Solbes, J. y Furió, C. (2013). Debates sobre cuestiones sociocientíficas: Una herramienta para aprender Física y Química. Textos, 64, 32-39.

Saxena A. (2019) Pedagogical Approaches to Ethical Issues in Biosciences. En Ethics in Science. Springer. https://doi.org/10.1007/978-981-32-9009-9_4

Shulman, L. (1986). Those who Understand: Knowledge Growth in Teaching. Educational Researcher, 15(2), 4-14. https://doi.org/10.3102/0013189X015002004

Sierra, C. E. (2007). Fortalezas epistemológicas y axiológicas de la ciencia ficción: un Potosí pedagógico mal aprovechado en la enseñanza y divulgación de las ciencias. Revista Eureka para la Enseñanza y Divulgación de las Ciencias, 4(1), 87 105. https://doi.org/10.25267/Rev_Eureka_ensen_divulg_cienc.2007.v4.i1.06

Sirin, S.; Rogers-Sirin, L.; Collins, B. (2010). A measure of cultural competence as an ethical responsibility: Quick-Racial and Ethical Sensitivity Test. Journal of Moral Education, 39(1), March 2010, pp. 49-64. https://doi. org/10.1080/03057240903528675

Solbes, J. y Traver, M. (2001). Resultados obtenidos introduciendo historia de la ciencia en las clases de física y química: mejora de la imagen de la ciencia y desarrollo de actitudes positivas. Enseñanza de las ciencias, 19(1), 151-162.

Solbes, J. y Traver, M. (1996). La utilización de la historia de las ciencias en la enseñanza de la física y la química. Enseñanza de las ciencias, 14(1), 103-112.

Tejeda Fernández, J. (2009). Competencias docentes. Profesorado. Revista de currículum y formación del profesorado, 13(2), 1-15. 
Timperley, H. y Alton-Lee, A. (2008). Reframing teacher professional learning: An alternative Policy Approach to strengthening valued outcomes for diverse learners. Review of Research in Education, 32, 1-45. https://doi. org/10.3102/0091732X07308968

Tuithof, H., Logtenberg, A., Bronckhorst, L., Van Drie, J., Dorsman, L. y Van Tartwijk, J. (2019). What do we know about the pedagogical content knowledge of history teachers: $A$ review of empirical research? Historical Encounters, 6(1), 72-95.

Universidad Nacional del Córdoba (s.f.). Maestría en Educación de las Ciencias Experimentales y la Tecnología, Universidad Nacional de Córdoba. http://www.mae.educacion. efn.uncor.edu/

Valcárcel, M. (2005) (Coord.) La preparación del profesorado universitario para la convergencia europea en educación superior. Educatio, 23, 209-213.

Vázquez, B., Jiménez, R., Mellado, V. (2007). El desarrollo profesional del profesorado como integración de la reflexión y la práctica. La hipótesis de la complejidad, Revista Eureka sobre Enseñanza y Divulgación de las Ciencias, 4(3), 372-393. https://doi. org/10.25267/Rev_Eureka_ensen_divulg_ cienc.2007.v4.i3.01

Viennot, L. y Décamp, N. (2018). Activation of a Critical Attitude In Prospective
Teachers: from Research Investigations to Guidelines for Teacher Education. Physical Review Physics Education Research, 14(1), 0101133 . https://doi.org/10.1103/PhysRevPhysEducRes. 14.010133

Wei, R., Darling Hammond, L. y Adamson, F. (2010). Professional Development in EEUU: Trends and Challenges. National Staff. Development Council. National Staff Development Council.

Zúñiga, A., Leiton, R. y Naranjo, J. (2014). Del sistema educativo tradicional hacia la formación por competencias: una mirada a los procesos de enseñanza aprendizaje de las ciencias en la educación secundaria de Mendoza Argentina y San José de Costa Rica. Revista Eureka sobre Enseñanza y Divulgación de las Ciencias, 17 (2), 145-159. https://doi.org/10.25267/Rev_Eureka_ensen_divulg_cienc.2014.v11.i2.03

\section{Para citar este artículo}

Cambra, I. y Lorenzo, M. (2021). La sensibilidad ética y el conocimiento didáctico del contenido de los profesores de ciencias experimentales. Tecné, Episteme y Didaxis: TED, (49), 199-218. https://doi.org/10.17227/ ted.num49-10000 
